\title{
A Novel Sparse Array for Localization of Mixed Near-Field and Far-Field Sources
}

\author{
Yinsheng Wang $\mathbb{D}^{\mathbb{D}}$, WeiJia Cui $(\mathbb{D})$, Yuxi Du $(\mathbb{D}$, , Bin Ba, and Fengtong Mei $(\mathbb{D}$ \\ National Digital Switching System Engineering \& Technological Research Center, Zhengzhou, Henan 450001, China \\ Correspondence should be addressed to Yinsheng Wang; yinshengw@163.com
}

Received 5 September 2021; Revised 16 November 2021; Accepted 25 November 2021; Published 20 December 2021

Academic Editor: Diego Masotti

Copyright (C) 2021 Yinsheng Wang et al. This is an open access article distributed under the Creative Commons Attribution License, which permits unrestricted use, distribution, and reproduction in any medium, provided the original work is properly cited.

\begin{abstract}
As we all know, nested array can obtain a larger array aperture and more degrees of freedom using fewer sensors. In this study, we not only designed an enhanced symmetric nested array (ESNA), which achieved more consecutive lags and more unique lags compared with a generalized nested array but also developed a special cumulant matrix, in the case of a given number of sensors, which can automatically generate the largest consecutive lags of the array. First, the direction-of-arrivals (DOAs) of mixed sources are estimated using the special cumulant matrix. Then, we can estimate the range of the near-field source in the mixed source using a one-dimensional spectral search through estimated DOAs, and in the mixed sources, the near-field and far-field sources are classified by bringing in the range parameter. The largest consecutive lags and composition method of ESNA are also given, under a given number of sensors.Our algorithm has moderate computation complexity, which provides a higher resolution and improves the parameters' estimation accuracy. Numerical simulation results demonstrate that the proposed array showed an outstanding performance under estimation accuracy and resolution ability for both DOA and range estimation compared with existing arrays of the same physical array sensors.
\end{abstract}

\section{Introduction}

Mixed source localization is an important problem in the field of array signal processing such as radar, sonar, and communications [1-5]. Therefore, to solve this problem, many algorithms have been proposed, such as the ESPRIT algorithm [6], the MUSIC algorithm [7], and so on. However, most of them focused on far-field (FF) assumption to solve the far-field problem [8]. When a source is located in the Fresnel region, which belongs to the near-field (NF) source, and the wavefront is no longer a plane wave but a spherical wave [9], the wavefront is composed of DOA and range [10].

Recently, many algorithms based on NF source localization have been developed to estimate DOA and the range [11-15]; however, the algorithms proposed above mainly focuses on NF or FF sources. In some other situations, for example, using microphone arrays to locate speakers and communications, mixed sources are received by an array
[16]. Fortunately, many algorithms have been proposed to deal with this situation. Based on the classical second-order statistics algorithms, an oblique projection algorithm was proposed by He et al. [17] and Zuo et al. [18] to eliminate the subspace of FF sources from the covariance matrix and obtain the corresponding subspace of NF sources. Corresponding NF and FF sources could be well separated; then with the NF DOA estimates, corresponding ranges parameters could be obtained via $1-D$ peak searching. However, when there are only FF sources, this method fails. To solve this problem, a RARE-based localization algorithm was proposed by Hua et al. [19]. This method eliminated the range parameters in the signal subspace, then obtained the NF DOA, and finally substituting NF DOA into a 1-D peak searching to obtain the range parameters.

It is generally known that high-order statistics (HOS) are getting more and more attention. HOS can not only increase the estimation accuracy but also extend an aperture of the given array. In addition, HOS is insensitive 
to Gaussian noise. A high-order MUSIC algorithm, proposed by Liang and Ding [20], DOA, and range are solved by constructing cumulant matrix, and it needed to construct two fourth-order cumulant matrices, which leads to high computation complexity. However, the methods mentioned above are all based on a uniform symmetric linear array to estimate the DOA and ranges of the mixed sources.

Noteworthy, in the case of coexistence of the near- and far-field sources, existing works mentioned above can be used. However, due to the use of uniform symmetric linear array, when physical array sensors are certain, consecutive lag numbers will be limited [21]. The problem of using fewer physical sensors to estimate more sources is becoming more and more important [22]. To solve this problem, many sparse linear arrays, such as nested arrays [23] and coprime arrays [24], have been proposed. The sparse array can produce a larger array aperture and more consecutive lags compared with uniform linear array with the same array sensors.

Some algorithms based on nested array for mixed sources localization have been developed. For example, using convolution neural networks to localization mixed sources [25], using exact spatial propagation geometry localization mixed sources [26], and components separation for localization and classification of mixed sources [27]. The methods mentioned above all used general nested arrays. Although the number of consecutive lags can be increased, they were not an optimal array.

According to the characteristics of a sparse array, an enhanced symmetric nested array (ESNA) and a novel algorithm for localization of mixed sources are proposed in this paper. By exploiting subarray partition method of the ESNA, one cumulant matrix that contains only DOA information to estimate the DOA of mixed sources was constructed using the conventional MUSIC method. Then, with the estimated DOA, the range parameters can be obtained by a 1-D peak searching. Compared with the existing arrays, ESNA not only exhibits a larger number of consecutive lags but also provides a larger array aperture under the given same sensor number. Compared with methods of Wang et al. in [28] and Akbar et al. in [10], due to use the ESNA and subarray partition method, we can obtain more continuous lags and better performance under the condition of the same sensor number.

In this paper, we designed the ESNA and a new algorithm to solve the mixed-field sources localization. Our main contributions are as follows:

(1) We proposed an enhanced symmetric nested array (ESNA). We also give the ESNA optimal array configuration parameters. Compared with uniform symmetric array, ESNA can provide higher spatial resolution and enhanced degrees of freedom with same sensors.

(2) Based on ESNA, we proposed a new algorithm, subarray partition, and compared with the existing similar algorithms, the complexity of this algorithm is greatly reduced.

(3) We verified the superiority of the proposed algorithm based on the ESNA in terms of mixed-field accuracy, resolution capacity, and many more DOFs and analyzed the range of ambiguity problem.

The remaining part of this paper is organized as follows: Section 2 introduces the signal model. Section 3 introduces the ESNA configuration and proposed algorithm. Section 4 provides the simulation results; the conclusions are given in Section 5 .

\section{Signal Model}

Consider $K$ narrow band and independent sources parameterized by $\left(\theta_{k}, r_{k}\right), k=1,2, \ldots, K$ impinge on the ESNA with $2 M+2 N+1$ sensors, from Figure 1, which consists of two symmetric sparse arrays with intersensor spacing $2 \mathrm{~N} d$ contains $2 \mathrm{M}$ sensors; and a symmetric uniform linear array with intersensor spacing $d$ contains $2 N-1$ sensors; and a left sensor, a right sensor with intersensor spacing $(2 \mathrm{~N}-1) d$ on both sides of the array. The distance between two sparse symmetric linear subarrays and a uniform linear array is $2 \mathrm{Nd}$. The sensors index can be expressed as $[-M-N,-M-N+1, \ldots, M+$ $N-1, M+N]$. We use set $W$ to represent the position of the sensors:

$$
W=\left[p_{-L} \cdot d, \ldots, p_{0} \cdot d, \ldots, p_{L} \cdot d\right],
$$

where $L=M+N$. The position of the $i$ th sensor is $p_{i} \cdot d, i$ $\in[-L, L]$. The value of $p_{i}$ is defined as

$$
p_{i}= \begin{cases}i, & |i| \leq N-1, \\ N-1+2 N(i-N+1), & N \leq i \leq M+N-1, \\ 1-N+2 N(i+N-1), & 1-M-N \leq i \leq-N, \\ N-2+2 N(M+1), & i=M+N, \\ 2-N-2 N(M+1), & i=-M-N .\end{cases}
$$

The reference point of the phase is the center of the array, that is, 0 array element is the reference point. When the $i$ th sensor receives a signal, it can be expressed as [20]

$$
x_{i}(t)=\sum_{k=1}^{K} s_{k}(t) e^{j \tau_{i k}}+n_{i}(t)
$$

where $s_{k}(t)$ represents the $k$ th narrowband source and $n_{i}(k)$ represents the additive Gaussian noise. In addition, $\tau_{i k}$ represents the propagation time delay of the $k$ th source arriving the $i$ th sensor relative to the 0 th sensor. If $k$ th source represents near-field one, $\tau_{i k}$ can be written in the following form:

$$
\tau_{i k}=i \gamma_{k}+i^{2} \phi_{k}
$$




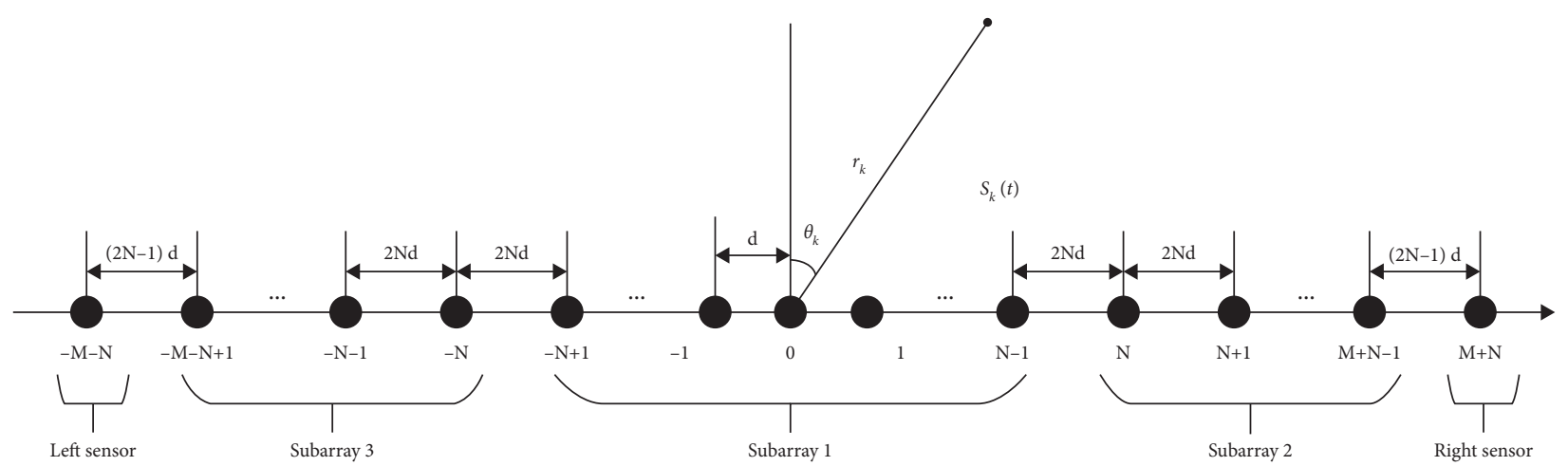

Figure 1: The ESNA geometry.

where $\gamma_{k}$ and $\phi_{k}$ are given by

$$
\begin{aligned}
& \gamma_{k}=-2 \pi \frac{d}{\lambda} \sin \left(\theta_{k}\right), \\
& \phi_{k}=\pi \frac{d^{2}}{\lambda r_{k}} \cos ^{2}\left(\theta_{k}\right),
\end{aligned}
$$

where $\theta_{k}$ and $r_{k}$ represent the azimuth DOA of $k$ th and range of the $k$ th near-field source. According to the definition, near-field source lies in the Fresnel zone $\left[0.62\left(D^{3} / \lambda\right)^{1 / 2}, 2 D^{2} / \lambda\right][14]$, where $D=2 p_{L} d$ is the array aperture. If the $k$ th source is a far-field one, then $r_{k} \longrightarrow \infty$, and $\tau_{i k}$ can be written the following form:

$$
\tau_{i k}=i \gamma_{k}
$$

where $\phi_{k} \approx 0$ due to beyond range of far-field source.

Assume that the $K_{1}$ sources are near-field sources and the rest of $K$ sources are far-field sources, then the vector form of equation (3) can be expressed as

$$
x(t)=A_{N} s_{N}(t)+A_{F} s_{F}(t)+n(t),
$$

where

$$
\begin{aligned}
x(t) & =\left[x_{-L}(t), \ldots, x_{0}(t), \ldots, x_{L}(t)\right]^{T}, \\
A_{N} & =\left[a\left(\theta_{1}, r_{1}\right), \ldots, a\left(\theta_{K_{1}}, r_{K_{1}}\right)\right], \\
A_{F} & =\left[a\left(\theta_{K_{1}+1}\right), \ldots, a\left(\theta_{K}\right)\right], \\
s_{N}(t) & =\left[s_{1}(t), \ldots, s_{K_{1}}(t)\right]^{T}, \\
s_{F}(t) & =\left[s_{K_{1}+1}(t), \ldots, s_{K}(t)\right]^{T},
\end{aligned}
$$

and

$$
n(t)=\left[n_{-L}(t), \ldots, n_{0}(t), \ldots, n_{L}(t)\right]^{T} .
$$

Note that the forms of steering vector $a\left(\theta_{k}, r_{k}\right)$ and $a\left(\theta_{k}\right)$ have the following form:

$$
\begin{aligned}
a\left(\theta_{k}, r_{k}\right) & =\left[e^{j\left(p_{-L} \gamma_{k}+p_{-L}^{2} \phi_{k}\right)}, \ldots, 1, \ldots, e^{j\left(p_{L} \gamma_{k}+p_{L}^{2} \phi_{k}\right)}\right]^{T}, \\
a\left(\theta_{k}\right) & =\left[e^{j\left(p_{-L} \gamma_{k}\right)}, \ldots, 1, \ldots, e^{j\left(p_{L} \gamma_{k}\right)}\right]^{T} .
\end{aligned}
$$

Some assumptions are required to hold in this paper:

(1) The source signals are narrowband, statistically mutually independent, and non-Gaussian processes with nonzero kurtosis

(2) The sensor noise is the additive white Gaussian process and independent of the source signals

(3) Using the ESNA array, in which the interelement spacing $d$ of the uniform linear array is set to be $(\lambda / 4)$

\section{Proposed Algorithm}

3.1. Analysis of ESNA in the Cumulant Domain. Cumulant has many advantages, it not only improves the estimation performance but also expands the array aperture [29-31]. In addition, cumulant can also provide an increased number of consecutive lags, which is insensitive to Gaussian noise.

Based on the assumptions, we define the fourth-order cumulant $\operatorname{cum}\left\{x_{m}(t), x_{n}^{*}(t), x_{\rho}^{*}(t), x_{q}(t)\right\}$ as

$$
\begin{aligned}
& \operatorname{cum}\left\{x_{m}(t), x_{n}^{*}(t), x_{\rho}^{*}(t), x_{q}(t)\right\} \\
&=\operatorname{cum}\left\{\sum_{k=1}^{K} s_{k}(t) e^{j\left(p_{m} \gamma_{k}+p_{m}^{2} \phi_{k}\right)},\left(\sum_{k=1}^{K} s_{k}(t) e^{j\left(p_{n} \gamma_{k}+p_{n}^{2} \phi_{k}\right)}\right)^{*},\right. \\
&\left.\cdot\left(\sum_{k=1}^{K} s_{k}(t) e^{j\left(p_{\rho} \gamma_{k}+p_{\rho}^{2} \phi_{k}\right)}\right)^{*}, \sum_{k=1}^{K} s_{k}(t) e^{j\left(p_{q} \gamma_{k}+p_{q}^{2} \phi_{k}\right)}\right\} \\
&= \sum_{k=1}^{K} c_{4, s_{k}} e^{j\left\{\left[\left(p_{m}-p_{n}\right)-\left(p_{\rho}-p_{q}\right)\right] \gamma_{k}+\left[\left(p_{m}^{2}-p_{n}^{2}\right)-\left(p_{\rho}^{2}-p_{q}^{2}\right)\right] \phi_{k}\right\}} \\
& \times \operatorname{cum}\left\{s_{k}(t), s_{k}^{*}(t), s_{k}^{*}(t), s_{k}(t)\right\} \\
&= \sum_{k=1}^{K} c_{4, s_{k}} e^{j\left\{\left[\left(p_{m}-p_{n}\right)-\left(p_{\rho}-p_{q}\right)\right] \gamma_{k}+\left[\left(p_{m}^{2}-p_{n}^{2}\right)-\left(p_{\rho}^{2}-p_{q}^{2}\right)\right] \phi_{k}\right\},}
\end{aligned}
$$

where $c_{4, s_{k}}=\operatorname{cum}\left\{s_{k}(t), s_{k}^{*}(t), s_{k}^{*}(t), s_{k}(t)\right\}$ is the kurtosis of the $k$ th signal and $m, n, \rho, q \in[-L, L]$.

In equation (11), we remove the $\phi_{k}$ term, while keeping the $\gamma_{k}$ term, it is assumed that $n=-m, q=-\rho$. Thus, equation (11) becomes 


$$
\begin{aligned}
\operatorname{cum} & \left\{x_{m}(t), x_{-m}^{*}(t), x_{\rho}^{*}(t), x_{-\rho}(t)\right\} \\
= & \sum_{k=1}^{K} c_{4, s_{k}} e^{j 2\left(p_{m}-p_{\rho}\right) \gamma_{k}} .
\end{aligned}
$$

Obviously, equation (12) only contains DOA parameters, which we can estimate DOA of all sources first by constructing a special cumulant matrix.

Proposition 1. Let $\mathbb{L}=\left\{l_{\rho} \mid-M-N \leq \rho \leq M+N\right\}$ represents the positions of the sensor in the ESNA array, and $\mathbb{L}_{c}$ $=\left\{l_{c} \mid l_{c}=p_{m}-p_{\rho}\right\}$ represents the lags in the ESNA. Based on the ESNA, we define the following facts to hold:

(a) There are $4 M N+8 N-3$ consecutive lags in the range $[-4 N-2 M N+2,4 N+2 M N-2]$

Proof (a): see Appendix.

(b) When $N=\lceil L+2 / 2\rceil$ and $M=\lfloor L+2 / 2\rfloor$, the largest consecutive lags can be obtained, where $\lceil\cdot\rceil$ and $\lfloor\cdot\rfloor$ are the round-towards infinite and round-towards zero

Proof: we define $f(N)=4 N M+8 N-3$, where $N=L-M . f^{\prime}(N)=4 L-8 N+8$ can be obtained by deriving $N$. Notably, $N$ is an integer, when $N=\lceil L+2 / 2\rceil$ or $M=\lfloor L-2 / 2\rfloor, f$ can get the maximum value.

As a comparison, four array configurations with $2 L+1$ sensors were considered: (1) symmetric nested array (SNA) [28], (2) symmetric double nested array (SDNA) [23], (3) improved symmetric nested array (ISNA) [10], and (4) the proposed enhanced symmetric nested array (ESNA). In Table 1, in the case of the same number of sensors, we compare the number of consecutive lags and the values of $M$ and $N$. Among all the nested arrays, the total sensors of the uniform linear array in the middle of a nested array are $2 N-1$, and the sparse array on both side of a uniform linear array is $2 M+2$. From Table 1 , it is obvious that the proposed ESNA has the maximum number of consecutive lags when the number of array sensors is the same. As the results show, the ESNA can offer more consecutive lags for DOA estimation in mixed source scenario.

In order to provide an intuitive impression of abovementioned four array configurations, herein, we give an example of 13 sensors and mark their positions in Figure 2. The red circles represent the lags of four virtual coarrays, and the blue crosses represent the holes for four virtual coarrays. The interval between positions is $d$.

3.2. DOA Estimation of All Sources. In this section, we construct a special cumulant, which can be used to eliminate the range parameter in the near-field, and then use the cumulant to estimate the DOA of the mixed field.

In the proposed algorithm, we use the subarray partition method to combine the sparse array and the uniform linear array in the ESNA form, and the corresponding cumulant output and the output cumulant are formed by the largest virtual consecutive lags.
Based on equation (11), first, let $m=M+N$, $p \in[1-N, \ldots, N-1]$, and the $(2 N-1) \times 1$ cumulant vector $c_{1}$ can be obtained, and its $(N-\rho)$ th element is given by

$$
\begin{aligned}
& c_{1}(N-\rho) \\
= & \operatorname{cum}\left\{x_{M+N}(t), x_{-M-N}^{*}(t), x_{\rho}^{*}(t), x_{-\rho}\right\} \\
= & \sum_{k=1}^{K} c_{4, s_{k}} e^{j 2((N-2)+2 N(M+1)-\rho) \gamma_{k}} .
\end{aligned}
$$

Similarly, since we are using a symmetric enhanced nested array, we can easily obtain a symmetrical part. Let $m=-M-N, \rho \in[1-N, \ldots, N-1]$, and we get another $(2 N-1) \times 1$ cumulant vector $\bar{c}_{1}$, and its $(N-\rho)$ th element is given by

$$
\begin{aligned}
& \bar{c}_{1}(N-\rho) \\
& =\operatorname{cum}\left\{x_{-M-N}(t), x_{M+N}^{*}(t), x_{-\rho}^{*}(t), x_{\rho}(t)\right\} \\
& =\sum_{k=1}^{K} c_{4, s_{k}} e^{j 2(-2 N-2 N(M+1)-\rho) \gamma_{k}} .
\end{aligned}
$$

Second, let $m \in[N, N+M-1], p \in[1-N, \ldots, N-1]$, we get another $M(2 N-1) \times 1$ cumulant vector $\mathbf{c}_{2 \_}$, and its $(2 N(m-N)+N-\rho)$ th element is given by

$$
\begin{aligned}
& c_{2 \_m}(2 N(m-N)+N-\rho) \\
& =\operatorname{cum}\left\{x_{m}(t), x_{-m}^{*}(t), x_{-\rho}^{*}(t), x_{\rho}(t)\right\} \\
& =\sum_{k=1}^{K} c_{4, s_{k}} e^{j 2((N-1)+2 N(m-N+1)-\rho) \gamma_{k}} .
\end{aligned}
$$

Similarly, let $m \in[N, N+M-2], \quad \rho=M+N$ and construct $(M-1) \times 1$ vector $\bar{c}_{2 \_}$, and its $(m-N+1)$ th element is given by

$$
\begin{aligned}
& \bar{c}_{2 \_m}(m-N+1) \\
& =\operatorname{cum}\left\{x_{M+N}(t), x_{-M-N}^{*}(t), x_{m}^{*}(t), x_{-m}(t)\right\} \\
& =\sum_{k=1}^{K} c_{4, s_{k}} e^{j 2(2 N(M+N-m)-1) \gamma_{k}} .
\end{aligned}
$$

Now, we construct the $(2 M N-1) \times 1$ vector $c_{2}$,

$$
c_{2}=\left[c_{2_{-} 1}, \bar{c}_{2_{-M+N-2}}, \ldots, c_{2_{-} M+N-2}, \bar{c}_{2_{-1}}, c_{2_{-} M+N-1}\right] \text {. }
$$

Similarly, since we are using a symmetric enhanced nested array, we can easily obtain a symmetrical part. Let $m \in[1-M-N,-N], \rho \in[1-N, \ldots, N-1]$, we get another $M(2 N-1) \times 1$ cumulant vector $c_{2}^{*} m$, and its $(-2 N(m+N)+N-\rho)$ th element is given by

$$
\begin{aligned}
& c_{2 \_m}^{*}(-2 N(m+N)+N-\rho) \\
& =\operatorname{cum}\left\{x_{m}(t), x_{-m}^{*}(t), x_{\rho}^{*}(t), x_{\rho}(t)\right\} \\
& =\sum_{k=1}^{K} c_{4, s_{k}} e^{j 2(1-N+2 N(m+N-1)-\rho) \gamma_{k}} .
\end{aligned}
$$


TABLE 1: Consecutive lags numbers for different arrays.

\begin{tabular}{|c|c|c|c|c|c|c|c|c|c|c|c|c|}
\hline \multirow{2}{*}{ Physical number } & \multicolumn{2}{|c|}{ SNA } & \multicolumn{2}{|c|}{ SDNA } & \multicolumn{2}{|c|}{ ISNA } & \multicolumn{2}{|c|}{ ESNA } & \multicolumn{4}{|c|}{ Maximum number of consecutive lags } \\
\hline & $N$ & $M$ & $N$ & $M$ & $N$ & $M$ & $N$ & $M$ & SNA & SDNA & ISNA & ESNA \\
\hline 7 & 1 & 1 & 2 & 2 & 3 & 1 & 3 & 0 & 7 & 15 & 19 & 21 \\
\hline 9 & 2 & 2 & 2 & 3 & 3 & 2 & 3 & 1 & 11 & 21 & 29 & 33 \\
\hline 11 & 2 & 2 & 3 & 3 & 4 & 2 & 4 & 1 & 17 & 29 & 41 & 45 \\
\hline 13 & 3 & 3 & 3 & 4 & 4 & 3 & 4 & 2 & 32 & 37 & 55 & 61 \\
\hline 15 & 3 & 3 & 4 & 4 & 5 & 3 & 5 & 2 & 31 & 47 & 71 & 77 \\
\hline 17 & 4 & 4 & 4 & 5 & 5 & 4 & 5 & 3 & 39 & 57 & 89 & 97 \\
\hline
\end{tabular}

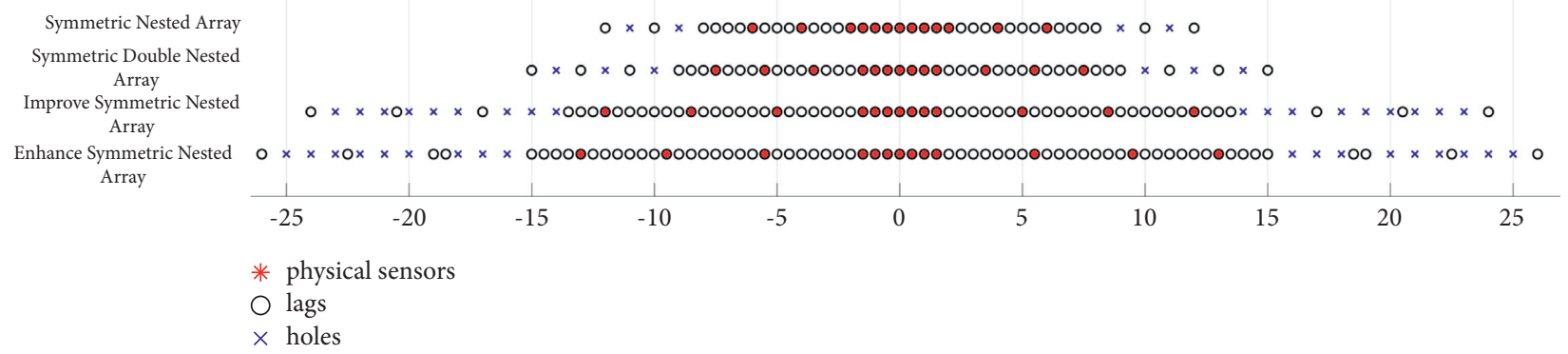

FIgURE 2: Sensor locations.

Similarly, let $m \in[2-M-N,-N], \rho=-M-N$ and construct $(M-1) \times 1$ vector $\bar{c}_{2_{-} m}^{*}$, and its $(m+N+2)$ th element is given by

$$
\begin{aligned}
& \bar{c}_{2 \_m}^{*}(m+N+2) \\
& =\operatorname{cum}\left\{x_{-M-N}(t), x_{M+N}^{*}(t), x_{m}^{*}(t), x_{-m}(t)\right\} \\
& =\sum_{k=1}^{K} c_{4, s_{k}} e^{j 2(1-2 N(M+N+m)) \gamma_{k}} .
\end{aligned}
$$

Now, we construct the $(2 M N-1) \times 1$ vector $\bar{c}_{2}$,

$$
\bar{c}_{2}=\left[c_{2_{-} 1}^{*}, \bar{c}_{2_{-M+N-2}}^{*}, \ldots, c_{2_{-} M+N-2}^{*}, \bar{c}_{2_{-1}}^{*}, c_{2_{-M+N-1}}^{*}\right] \text {. }
$$

Third, let $m \in[1-N, N-1], \rho=0$ and construct the $(2 N-1) \times 1$ vector $c_{3}$, and its $m$ th element is given by

$$
\begin{aligned}
& c_{3}(m+N) \\
& =\operatorname{cum}\left\{x_{m}(t), x_{-m}^{*}(t), x_{0}^{*}(t), x_{0}(t)\right\} \\
& =\sum_{k=1}^{K} c_{4, s_{k}} e^{j 2 m \gamma_{k}} .
\end{aligned}
$$

Fourth, let $m=N-1, \rho \in[1-N,-1]$, we get another $(N-1) \times 1$ cumulant vector $c_{4}$, and its $(N+\rho)$ th element is given by

$$
\begin{aligned}
& c_{4}(N+\rho) \\
& =\operatorname{cum}\left\{x_{m}(t), x_{-m}^{*}(t), x_{\rho}^{*}(t), x_{\rho}(t)\right\} \\
& =\sum_{k=1}^{K} c_{4, s_{k}} e^{j 2(m-\rho) \gamma_{k}} .
\end{aligned}
$$

Similarly, since we are using a symmetric enhanced nested array, we can easily obtain a symmetrical part, let $m=1-N, \rho \in[1, N-1]$, we construct the $(N-1) \times 1$ vector $\bar{c}_{4}$, and its $\rho$ th element is given by

$$
\begin{aligned}
& \bar{c}_{4}(\rho) \\
& =\operatorname{cum}\left\{x_{m}(t), x_{-m}^{*}(t), x_{\rho}^{*}(t), x_{\rho}(t)\right\} \\
& =\sum_{k=1}^{K} c_{4, s_{k}} e^{j 2(m-\rho) \gamma_{k}} .
\end{aligned}
$$

Fifth, let $m=M+N, \rho=M+N-1$ and $m=-M-N$, $\rho=1-M-N$, and we construct the last two cumulants $c_{5}$ and $\bar{c}_{5}$,

$$
\begin{aligned}
& c_{5} \\
& =\operatorname{cum}\left\{x_{M+N}(t), x_{-M-N}^{*}(t), x_{M+N-1}^{*}(t), x_{1-M-N}(t)\right\} \\
& =\sum_{k=1}^{K} c_{4, s_{k}} e^{j 2(2 N-1) \gamma_{k}},
\end{aligned}
$$

and

$$
\begin{aligned}
& \bar{c}_{5} \\
& =\operatorname{cum}\left\{x_{-M-N}(t), x_{M+N}^{*}(t), x_{1-M-N}^{*}(t), x_{M+N-1}(t)\right\} \\
& =\sum_{k=1}^{K} c_{4, s_{k}} e^{j 2(1-2 N) \gamma_{k}} .
\end{aligned}
$$

Finally, let $m=M+N-1, \rho=-N$, we construct $c_{\text {end }}$, and it is given by

$$
\begin{aligned}
& c_{\text {end }} \\
& =\operatorname{cum}\left\{x_{M+N-1}(t), x_{1-M-N}^{*}(t), x_{-N}^{*}(t), x_{N}(t)\right\} \\
& =\sum_{k=1}^{K} c_{4, s_{k}} e^{j 2(4 N+2 N M-2) \gamma_{k}} .
\end{aligned}
$$


Similarly, since we are using a symmetric enhanced nested array, we can easily obtain a symmetrical part. Let $m=1-M-N, \rho=N$, we construct $\bar{c}_{\text {end }}$, and it is given by

$$
\begin{aligned}
& \bar{c}_{\text {end }} \\
& =\operatorname{cum}\left\{x_{1-M-N}(t), x_{M+N-1}^{*}(t), x_{N}^{*}(t), x_{-N}(t)\right\} \\
& =\sum_{k=1}^{K} c_{4, s_{k}} e^{j 2(4 N+2 N M-2) \gamma_{k}} .
\end{aligned}
$$

Now, we combine the above vectors to form a $2(4 N+$ $2 M N-1)-1 \times 1$ vector $c$, which is expressed as

$$
c=\left[\bar{c}_{\text {end }}, \bar{c}_{1}^{T}, \bar{c}_{2}^{T}, \bar{c}_{5}, \bar{c}_{4}^{T}, c_{3}^{T}, c_{4}^{T}, c_{5}, c_{2}^{T}, c_{1}^{T}, c_{\text {end }}\right]^{T} .
$$

A Toeplitz matrix $C$ is constructed through the vector $c$, and matrix $C$ is $(4 N+2 M N-1) \times(4 N+2 M N-1)$, whose $m$ th column of $C$ consists of the $((4 N+2 M N-1)+1-m)$ th to $(2(4 N+2 M N-1)+1-m)$ th element of $c$. The Toeplitz matrix $C$ can be expressed in a compact form as

$$
\begin{aligned}
C & =A(\theta) C_{s} A^{H}(\theta), \\
C_{s} & =\operatorname{diag}\left[c_{4 s_{1}}, \ldots, c_{4 s_{K}}\right], \\
A(\theta) & =\left[a\left(\theta_{1}\right), \ldots, a\left(\theta_{K}\right)\right],
\end{aligned}
$$

and

$$
a\left(\theta_{k}\right)=\left[1, e^{j 2 \gamma_{1}}, \ldots, e^{j 2(4 N+2 M N-2) \gamma_{k}}\right]^{T}
$$

Since the sources signals have nonzero fourth-order cumulant, the matrix $C$ is full rank. Therefore, we estimate the common $\theta$ of mixed sources by performing the eigenvalue decomposition of $C$.

Based on the EVD of $C$, using the MUSIC algorithm [6] to estimate DOA and we can get the following formula:

$$
\theta_{k}=\min a(\theta){ }^{H} U_{n} U_{n}^{H} a(\theta), \quad k=1,2, \ldots, K,
$$

where $U_{n}$ represents the noise subspace of $C$.

3.3. Range Estimation and Source Type Classification. Once $\bar{\theta}_{k}, k=1, \ldots, K$ is estimated, then we can estimate $r_{k}$. When we get the convariance matrix $R=E\left\{x(t) x^{H}(t)\right\}$, perform eigen-decomposition on it, and we can get

$$
R=\bar{U}_{s} \bar{V}_{s} \bar{U}_{s}^{H}+\bar{U}_{n} \bar{V}_{n} \bar{U}_{n}^{H},
$$

where $\bar{U}_{n}$ spans the noise subspace, which contains $(4 N+$ $2 M N-1-K)$ eigenvectors. By taking the estimated $\bar{\theta}_{k}$ into $a\left(\theta_{k}, r\right)$, the $r_{k}$ can be obtained by the following:

$$
r_{k}=\min a\left(\theta_{k}, r\right) \bar{U}_{n} \bar{U}_{n}^{H} a^{H}\left(\theta_{k}, r\right), \quad k=1, \ldots, K .
$$

Obviously, $K$ peaks can be obtained by searching in the 2-D MUSIC spectrum [11]. When the range $r_{n}$ lies in the Fresnel region $r_{n} \in\left[0.62\left(D^{3} / \lambda\right)^{1 / 2}, 2 D^{2} / \lambda\right]$, through spectrum search, we can get $K 1$ near-field sources, when the range $r_{n}$ exceeds the Fresnel region $r_{f}=\infty, K-K 1$ far-field sources we can get through the spectrum search [28].
Finally, $\bar{\theta}_{k}$ and $\bar{r}_{k}$ are paired one by one, and no operation is required.

3.4. Ambiguity Analysis of Range Estimation. In this part, the ambiguity of range needs us to discuss because the ranges of near-field are estimated by ESNA, where some intersensor spacings are more than a half wavelength. In $[21,28,32]$, the use of a sparse array to estimate ambiguity problem of range of a given DOA has been discussed. Our analysis is similar to that of [28]. We analyze the ambiguity of the range under the ESNA.

Like [28], according to equation (33), the steering vector $a\left(\theta_{k}, r_{k}\right)$ contains the range parameter $r_{k}$. If $r_{k}$ is unique, then there is no ambiguity in the steering vector $a\left(\theta_{k}, r_{k}\right)$.

Similarly, suppose that $a\left(\theta_{k}, r_{k}\right)$ is ambiguous, from equations (3), (4), and (6), we have

$$
\frac{\pi d^{2} p_{m}^{2} \cos ^{2}\left(\theta_{k}\right)}{\lambda r_{k}}=\frac{\pi d^{2} p_{m}^{2} \cos ^{2}\left(\theta_{k}\right)}{\lambda \bar{r}_{k}}+h 2 \pi,
$$

where $r_{k}$ denotes the true range and the $\bar{r}_{k}$ denotes the false range, $r_{k}, \bar{r}_{k} \in\left[0.62\left(D^{3} / \lambda\right)^{1 / 2}, 2 D^{2} / \lambda\right]$. When the integer $|h| \geq 1$, the range must be ambiguous. Thus, equation (34) can be transformed into

$$
p_{m}^{2} \geq \frac{2 \lambda}{d^{2} \cos ^{2}\left(\theta_{k}\right)} \cdot \frac{1}{\left|\left(1 / r_{k}\right)-\left(1 / \bar{r}_{k}\right)\right|} .
$$

By minimizing the right-side part of equation (35), we can obtain the maximum range of $p_{m}^{2}$. Let $\cos ^{2}\left(\theta_{k}\right)=1$, $r_{k}=0.62\left(D^{3} / \lambda\right)^{1 / 2}$, and $\bar{r}_{k}=\infty$, then we have

$$
p_{m}^{2} \geq \frac{2 \lambda}{d^{2}} 0.62\left(\frac{D^{3}}{\lambda}\right)^{1 / 2}
$$

If we take the array aperture equal to $\lambda$ and $d$ equal to $\lambda / 4$, we can get

$$
\left|p_{m}\right| \geq 4.45 \text {. }
$$

which implies that when $p_{m} \geq 5$ or $p_{m} \leq-5$, the $m$ th element of the steering vector $a\left(\theta_{k}, r\right)$ is not unique. However, when $D=\lambda$ and $d=\lambda / 4,\left|p_{m}\right|$ is less than 5 in the ESNA. Thus, when $-4 \leq\left|p_{m}\right| \leq 4$, the $m$ th element of $a\left(\theta_{k}, r\right)$ is unique.

Consequently, through the analysis of the above, we can obtain that the range $r_{k}$ is unique through the DOA estimation $\theta_{k}$ from equation (33).

3.5. Computational Complexity. Regarding the computational complexity, the main computation load of the proposed algorithm includes the cumulant matrix and covariance matrices that need to be constructed. For $2 L+1$ sensors and $L=M+N$. For $M=N$, the multiplications corresponding to the proposed method are $\mathcal{O}\{9(2) 4 M+$ $\left.\left.\left.\left.2 M^{2}-1\right)-1\right) T+(4 M+1)^{2} T\right)\right\}$, where $T$ is the snapshot number and the multiplications corresponding to the highorder MUSIC mixed-order MUSIC [20] are $\mathcal{O}\{9(4 M+$ $\left.\left.1)^{2} T+9(8 M+1)^{2} T\right)\right\}$ and mixed-order MUSIC [21] are $\left.\mathcal{O}\left\{9\left(2(4 M+1)^{2}+1\right) T+(4 M+1)^{2}\right)\right\}$, respectively. Hence, 
the complexity of the proposed algorithm is the lowest compared with the above two classical algorithms.

\section{Simulation Results}

In the last section, the performance of the proposed array is evaluated by simulation results, which are compared with the other three array geometries, SNA [28], SDNA [29], and ISNA [21], and we also compared with HOS MUSIC [20] and mixed-order MUSIC [21] algorithms to demonstrate the performance of the proposed algorithm. The total number of elements is $Q=13$. The sources signal is $e^{j \zeta_{t}}$, where the phases $\zeta_{t}$ are uniformly distributed in $[0,2 \pi]$. However, the four arrays have different array apertures; therefore, they have different Fresnel regions. Here, taken the common Fresnel region for NF region $[29 \lambda<r<70 \lambda]$ to all arrays. The performance of the four arrays and proposed algorithm are evaluated through the root-mean-square error (RMSE) curve, and the number of Monte-Carlo trials is $N_{s}=500$.

$$
\mathrm{RMSE}=\sqrt{\frac{1}{N_{s} K} \sum_{k=1}^{K} \sum_{n_{s}=1}^{N_{s}}\left(\bar{\alpha}_{k}^{(m)}-\alpha_{k}\right)^{2}}
$$

where $\alpha_{k}$ represents the DOA $\theta_{k}$ or the range $r_{k}$ and $\bar{\alpha}_{k}$ is the estimate of $\alpha_{k}$ for the $m$ th trial. The resolution probability can be defined as if the estimated DOA of two sources satisfies $\left|\bar{\theta}_{k}-\theta_{k}\right|<\varepsilon / 2$, where $\varepsilon$ is the difference between the two angles, it is considered that it can successfully distinguish two angles.

4.1. Experiment 1. In the first simulation, we used two NF sources located at $\left(20^{\circ}, 30 \lambda\right),\left(50^{\circ}, 35 \lambda\right)$ and two FF sources located at $\left(-40^{\circ}, \infty\right),\left(20^{\circ}, \infty\right)$, respectively. The SNR is fixed at $15 \mathrm{~dB}$, and the number of snapshots is set at 2000 . The spatial spectrum of mixed sources is drawn in Figure 3. We can observe from Figure 3(a) that a total of four obvious peaks are estimated, two of which are near-field sources and two are far-field sources. We can also see from Figure 3(b) that there aretwo peaks located in the NF region and another two peaks in the FF region. According to Figure 3, the proposed algorithm can effectively distinguish the near-field and far-field in the mixed field.

4.2. Experiment 2. In the second simulation, we compare the probability of arrays for two closely spaced sources against SNR in Figure 4. In Figure 4(a), two pure NF sources are located at $\left(40^{\circ}, 31 \lambda\right),\left(44^{\circ}, 32 \lambda\right)$, and the angle interval $\varepsilon$ of the two is $4^{\circ}$. In Figure 4(b), two pure FF sources are located at $\left(-20^{\circ}, \infty\right),\left(-16^{\circ}, \infty\right)$, and the angle interval $\varepsilon$ of the two is $4^{\circ}$. In Figure 4(c), two mixed sources and one NF source are located at $\left(34^{\circ}, 31 \lambda\right)$, another FF source is located at $\left(30^{\circ}, \infty\right)$, and the angle interval $\varepsilon$ of the two is also $4^{\circ}$. When $T=2000$ snapshots, and SNR $\in[-6,24] \mathrm{dB}$. From the simulation result, compared with other arrays, the proposed ESNA can obtain better estimation accuracy under the same conditions, and it can successfully distinguish the close sources at lower SNR.
4.3. Experiment 3. In the third simulation, we used two pure near-field sources located at $\left(40^{\circ}, 31 \lambda\right)$ and $\left(50^{\circ}, 32 \lambda\right)$, respectively:

(1) RMSE versus SNR: when snapshots $=2000$, and SNR $\in[-5,30] \mathrm{dB}$, from Figure 5, we can see the RMSE curves of DOA and range. As can be seen from Figures 5(a) and 5(b), in all SNRs, the ESNA can provide better estimation accuracy than the other arrays. As is expected, when the SNR increases, the RMSE curves of DOA and range show a downward trend.

(2) RMSE versus snapshots: when $\mathrm{SNR}=15 \mathrm{~dB}$, and snapshots $\in[50,2000]$, from Figure 6, we can see the RMSE curves of DOA and range. From Figures 6(a) and 6(b), we can see that the proposed algorithm still has a better performance than the other algorithms for all available snapshots. This is also because the ESNA can obtain more consecutive lags compared with other arrays. As is expected, with the increase of snapshot, the RMSE curves show a downward trend.

4.4. Experiment 4. In the fourth simulation, we used mixed sources. One near-field source is located at $\left(30^{\circ}, 30 \lambda\right)$, and another far-field source is located at $\left(-10^{\circ}, \infty\right)$ :

(1) RMSE versus SNR: when snapshots $=2000$, and SNR $\in[-5,30] d B$, from Figure 7 , we can see the RMSE curves of DOA and range about mixed sources (range estimations are not performed for far-field source). As can be seen from Figures 7(a) and 7(b), in all SNRs, the ESNA can obtain better estimation accuracy than the other arrays. As is expected, when the SNR increases, the RMSE curves of DOA and range show a downward trend.

(2) RMSE versus snapshots: when $\mathrm{SNR}=15 \mathrm{~dB}$, and snapshots $\in[50,2000]$, from Figure 8 , we can see the RMSE curves of DOA and range. As can be seen from Figures 8(a) and 8(b), in all snapshots, the ESNA can obtain better estimation accuracy than the other arrays. As is expected, with the increase of snapshot, the RMSE of DOA and range show a downward trend.

4.5. Experiment 5. In the fifth simulation, we used to deal with two pure far-field sources. Two FF sources are located at $\left(40^{\circ}, \infty\right),\left(50^{\circ}, \infty\right)$, respectively:

(1) RMSE versus SNR: when snapshots $=2000$, and SNR $\in[-5,30] d B$, from Figure 9, we can see the RMSE curves of DOA and range. As can be seen from Figure 9(a), in all SNRs, the ESNA can obtain better estimation accuracy than the other arrays. This is because the ESNA can obtain more consecutive lags compared with other arrays. As is expected, when the SNR increases, the RMSE of DOA and range show a downward trend. 


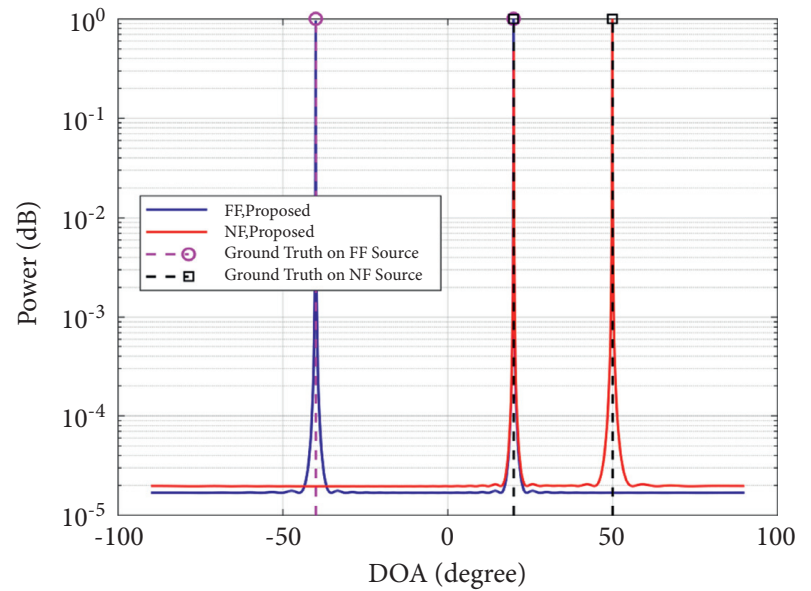

(a)

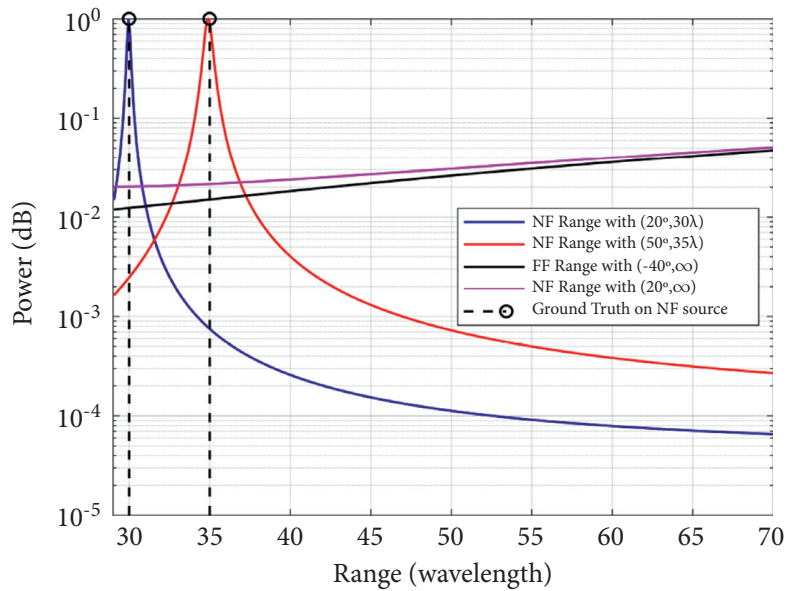

(b)

FIGURE 3: Spatial spectrum of mixed sources. SNR $=15 \mathrm{~dB}$ and $T=2000$ snapshots. (a) Spatial spectrum of the NF and FF estimators. (b) Spatial spectrum of range estimators.
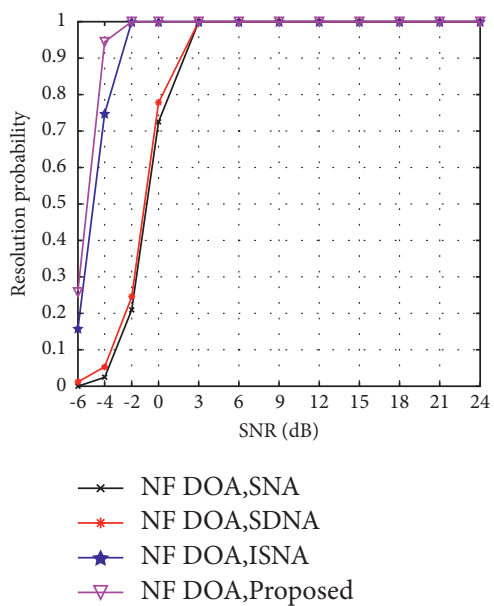

(a)
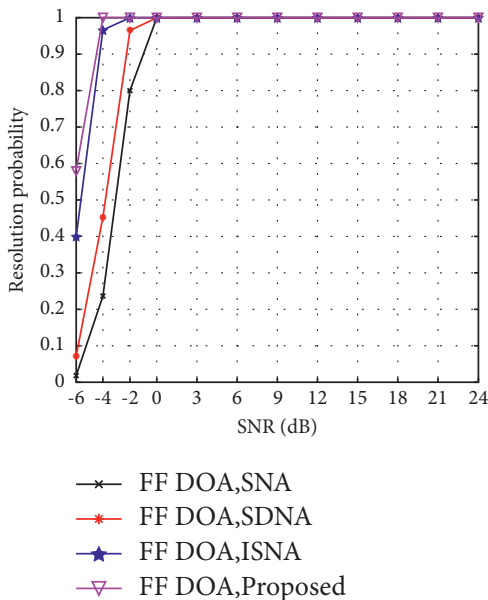

(b)
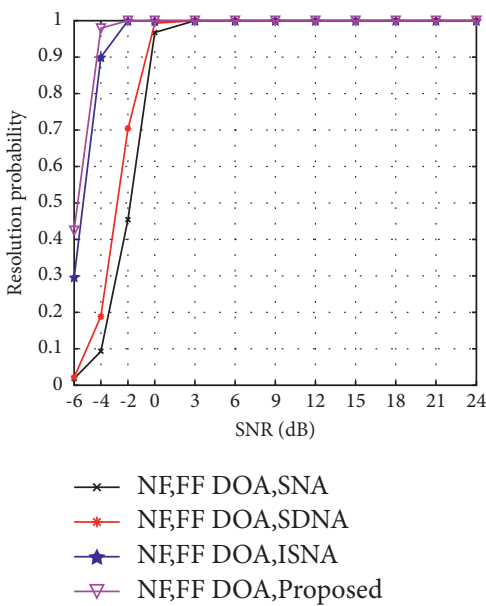

(c)

FIGURE 4: Probability versus SNR for closely spaced sources. (a) Near-field. (b) Far-field. (c) Mixed sources.

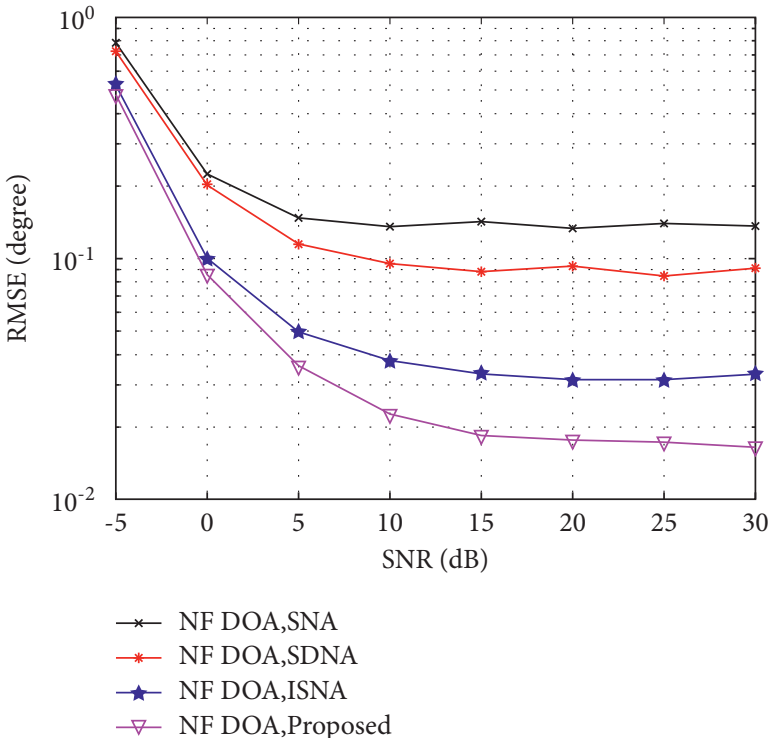

(a)

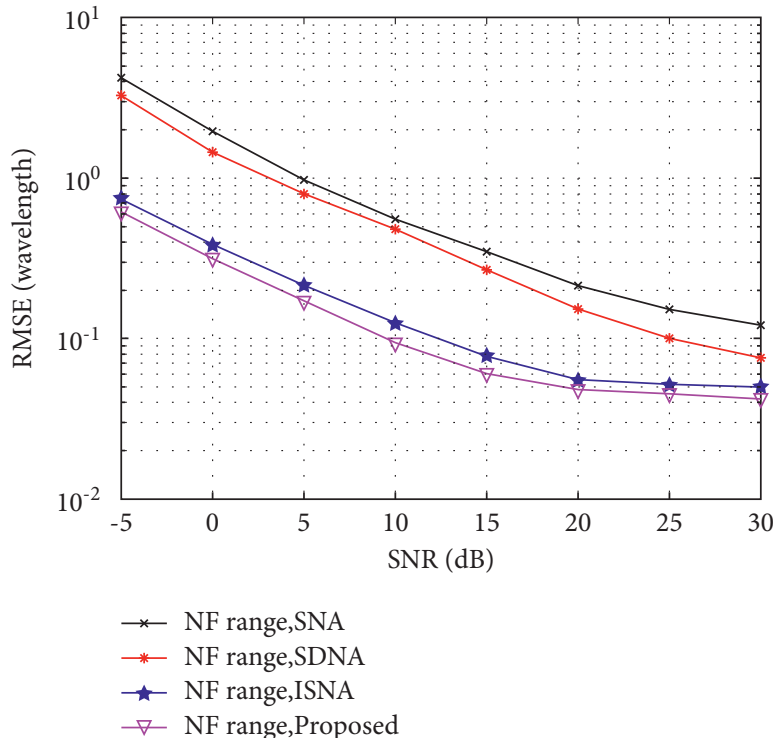

(b)

Figure 5: RMSE curves of DOA and range estimation versus SNR. (a) DOA estimations for near-field. (b) Range estimations for near-field. 


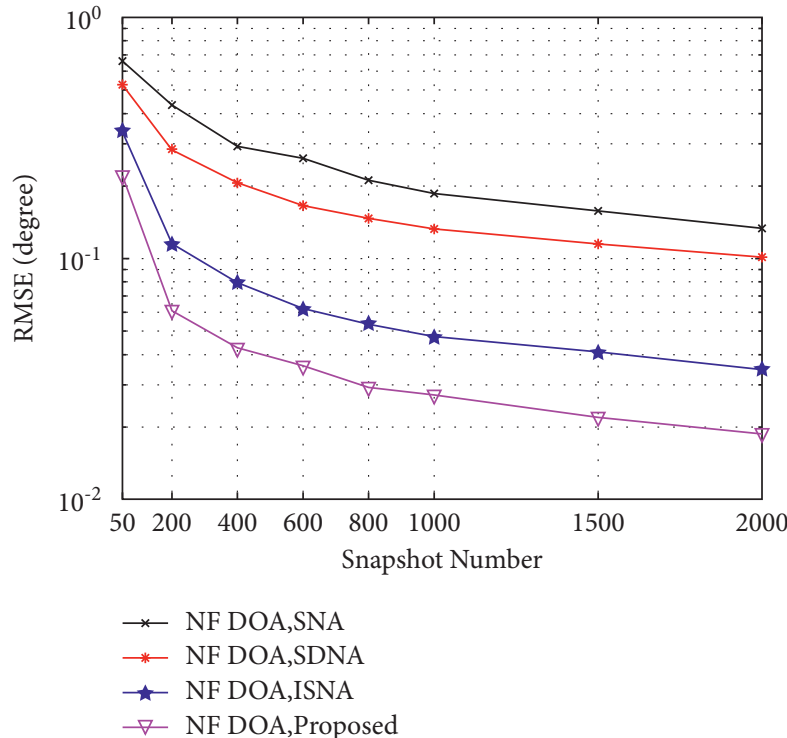

(a)

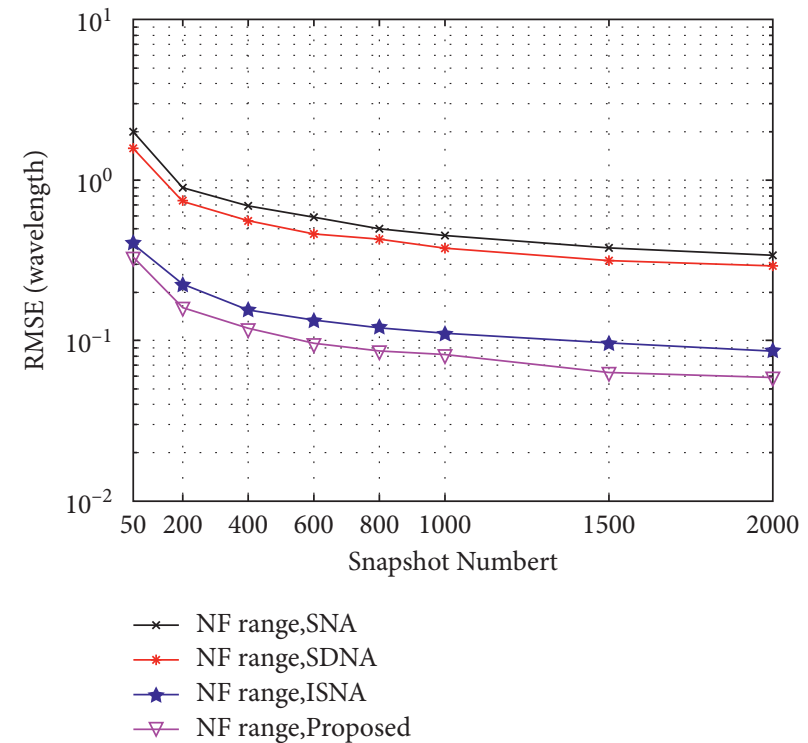

(b)

FIGURE 6: RMSE curves of DOA and range estimation versus snapshots. (a) DOA estimations for near-field. (b) Range estimations for near-field.

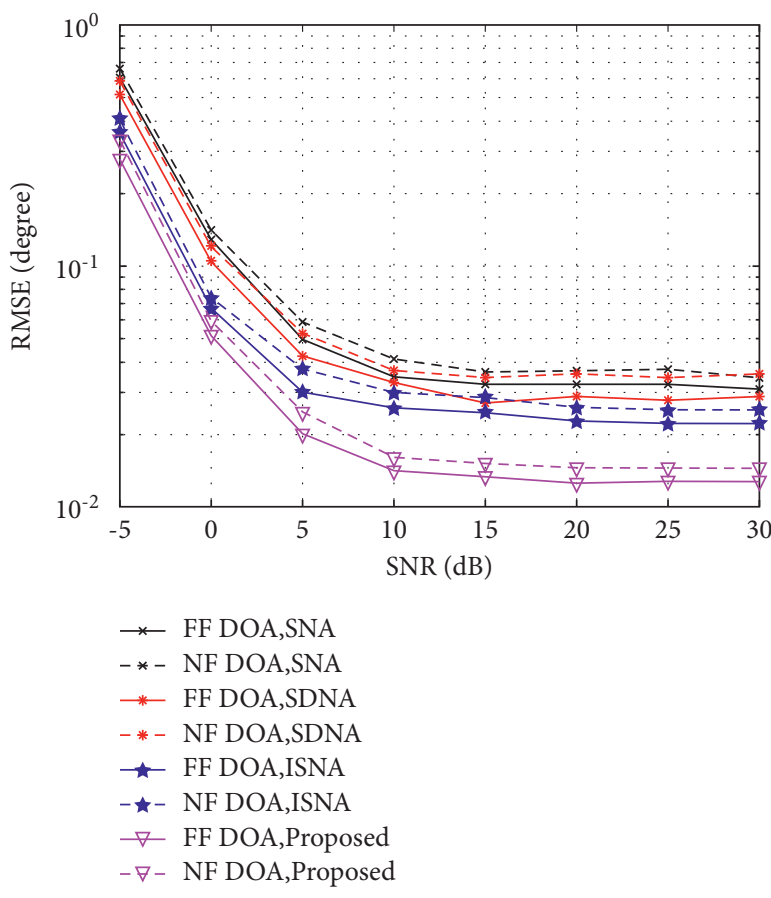

(a)

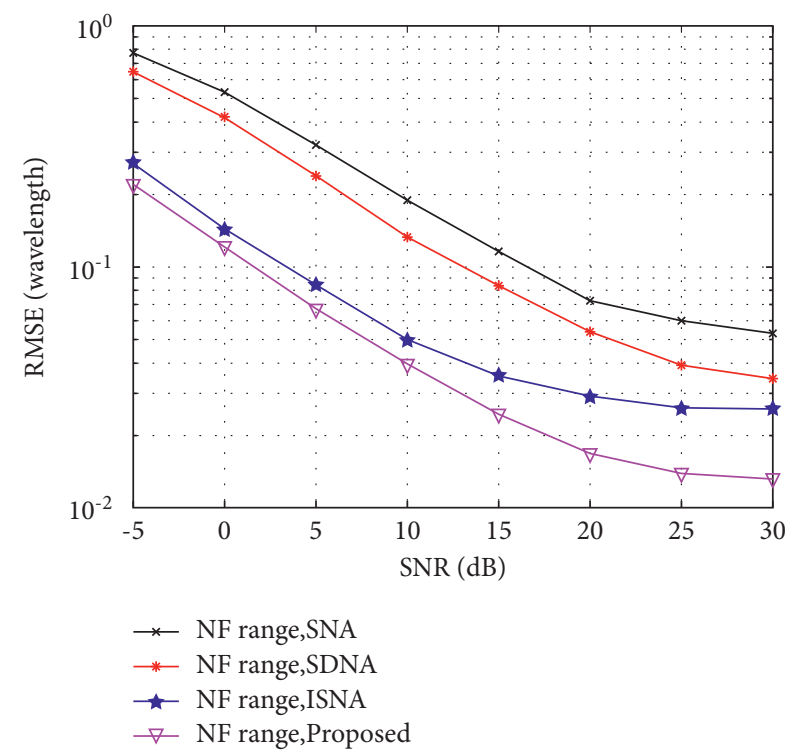

(b)

FIgURE 7: RMSE curves of DOA and range estimation versus SNR. (a) DOA estimations for NF and FF. (b) Range estimations for NF.

(2) RMSE versus snapshots: when $\mathrm{SNR}=15 \mathrm{~dB}$, and snapshots $\epsilon[50,2000]$, from Figure 9, we can see the RMSE curves of DOA and range. As can be seen from Figure 9(b), we can see that the proposed algorithm still has a better performance than the other algorithms for all available snapshots. This is also because the ESNA can obtain more consecutive lags compared with other arrays. As is expected, with the increase of snapshot, the RMSE of DOA and range show a downward trend.

4.6. Experiment 6. In the last simulation, we present simulations to prove that our proposed algorithm outperforms HOS MUSIC and mixed-order MUSIC algorithms. We used mixed sources. One near-field source is 


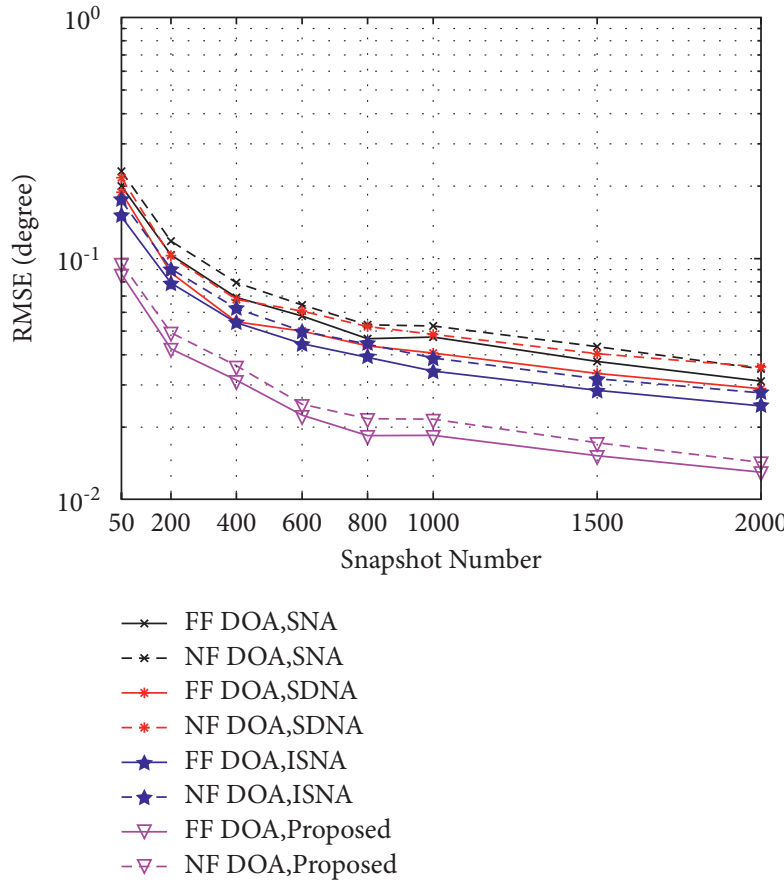

(a)

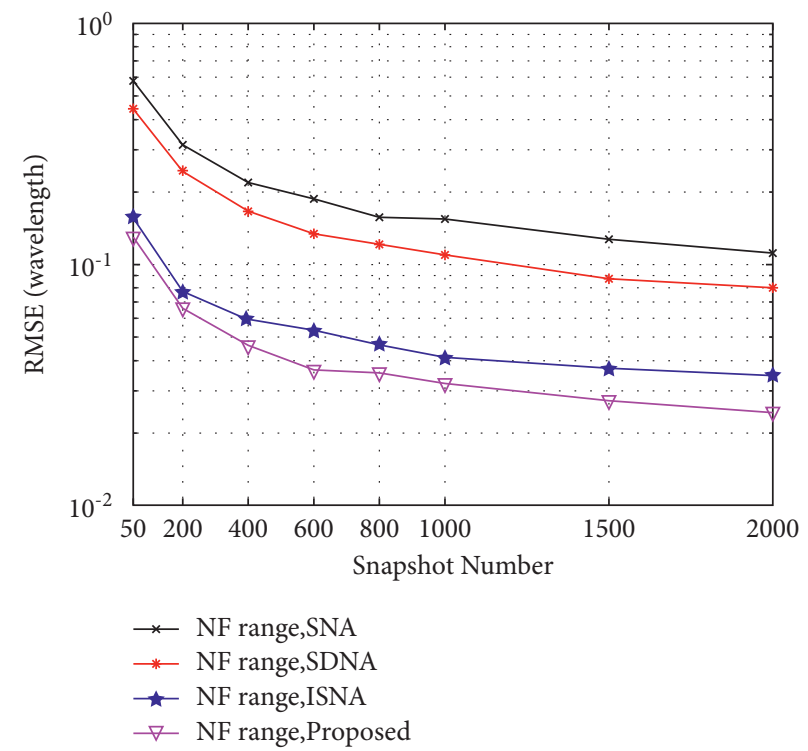

Figure 8: RMSE curves of DOA and range estimation versus snapshots. (a) DOA estimations for NF and FF. (b) Range estimations for NF.

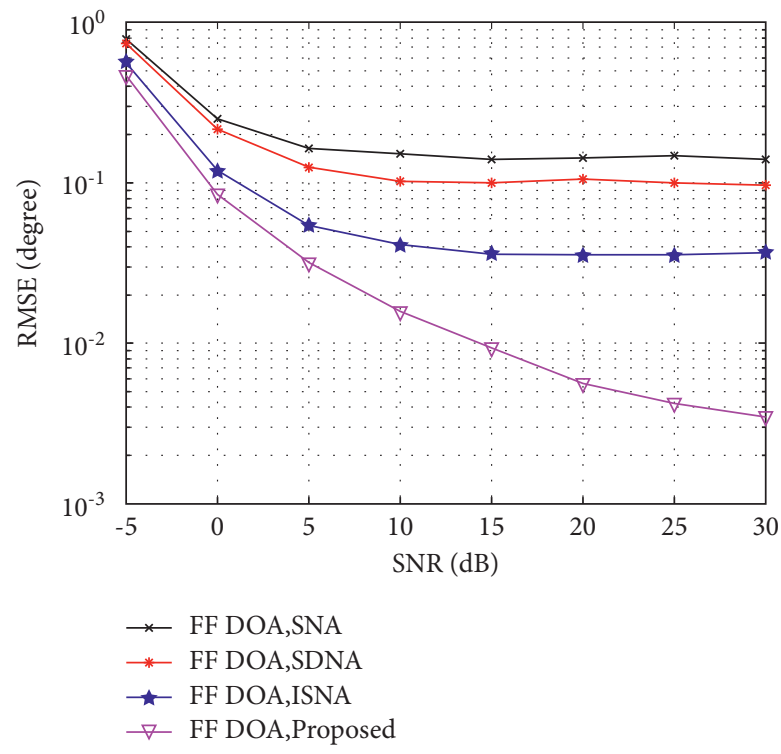

(a)

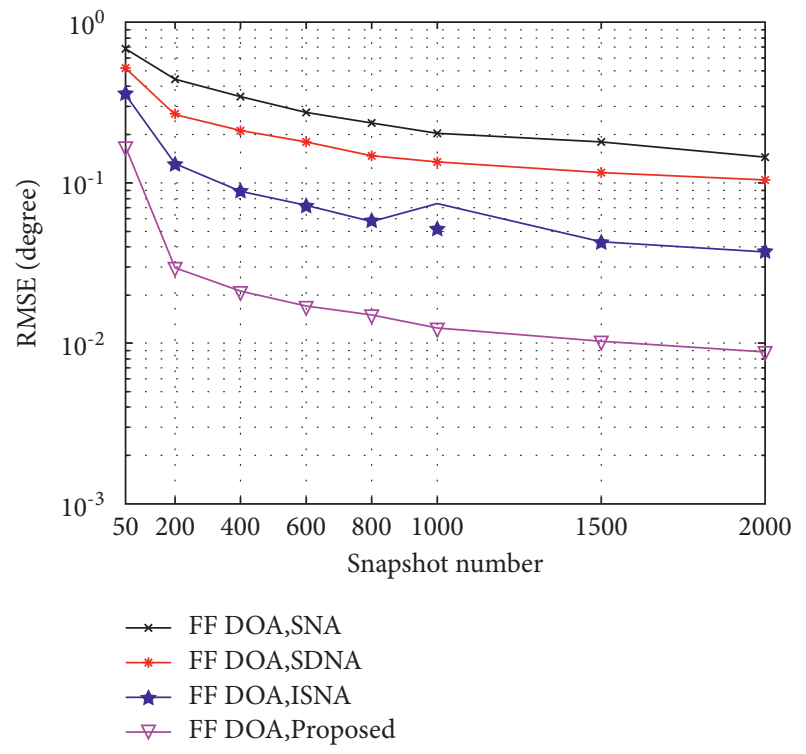

(b)

FIGURE 9: RMSE curves of DOA estimation for pure FF versus SNR and snapshots. (a) DOA estimations for pure FF. (b) DOA estimations for pure FF.

located at $\left(20^{\circ}, 31 \lambda\right)$, and another far-field source is located at $\left(30^{\circ}, \infty\right)$ :

(1) RMSE versus SNR: when snapshots $=2000$, and SNR $\in[-5,30] d B$, from Figure 10, we can see the RMSE curves of DOA (range estimation is not performed for mixed-field source). As can be seen from Figure 10(a), in all SNRs, the proposed algorithm can obtain better estimation accuracy than the other algorithms. As is expected, when the SNR increases, the RMSE curves of DOA show a downward trend.

(2) RMSE versus snapshots: when $\mathrm{SNR}=15 \mathrm{~dB}$, and snapshots $\in[50,2000]$, from Figure 10 , we can see the RMSE curves of DOA (range estimation is not performed for mixed-field source). As can be seen 


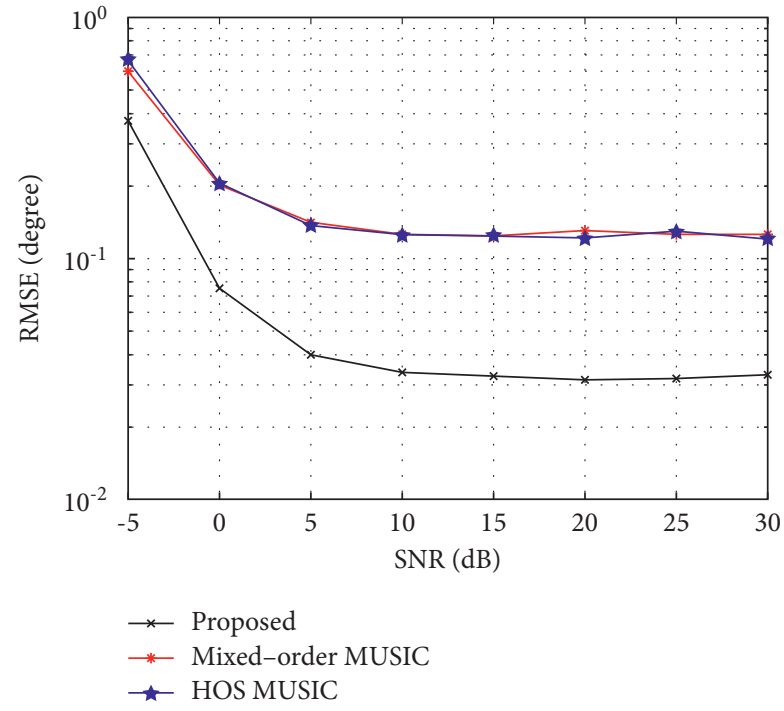

(a)

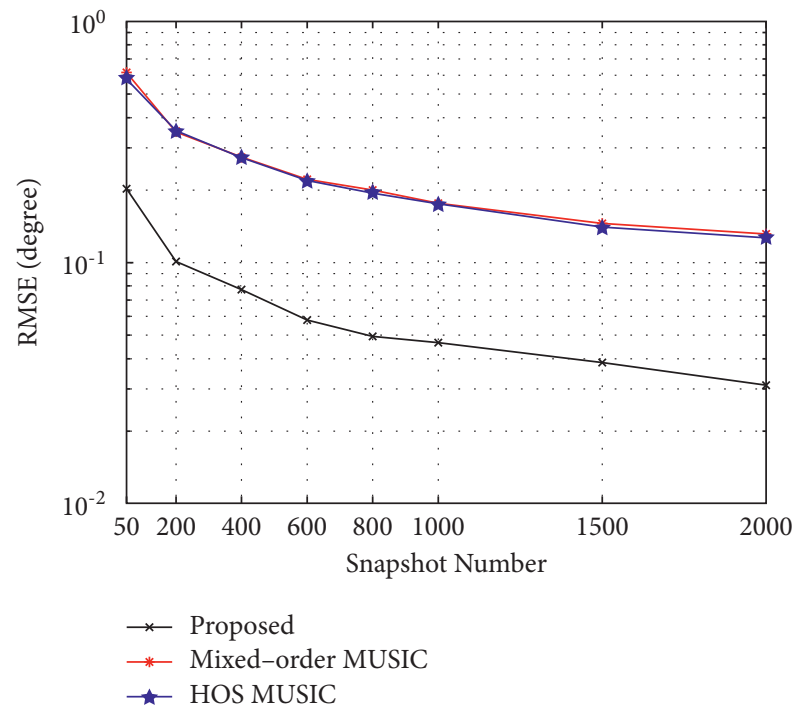

(b)

FIGURE 10: RMSE curves of DOA estimation for pure FF versus SNR and snapshots. (a) DOA estimations for SNR. (b) DOA estimations for snapshots.

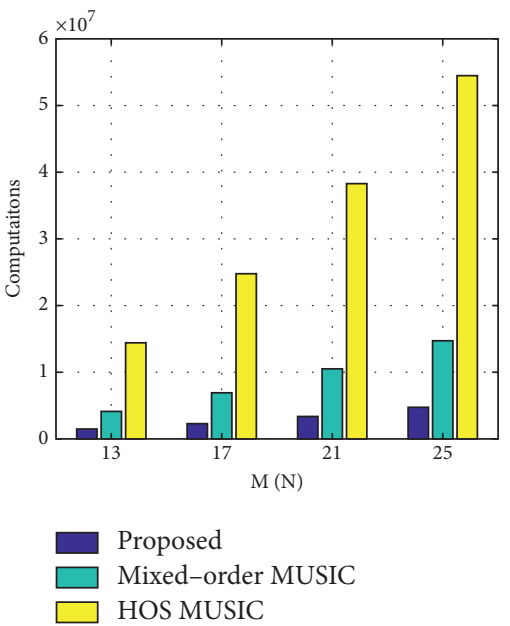

(a)

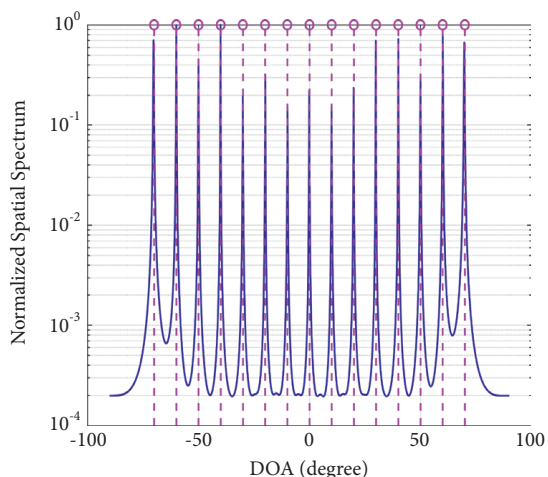

DOA (degree)

(b)

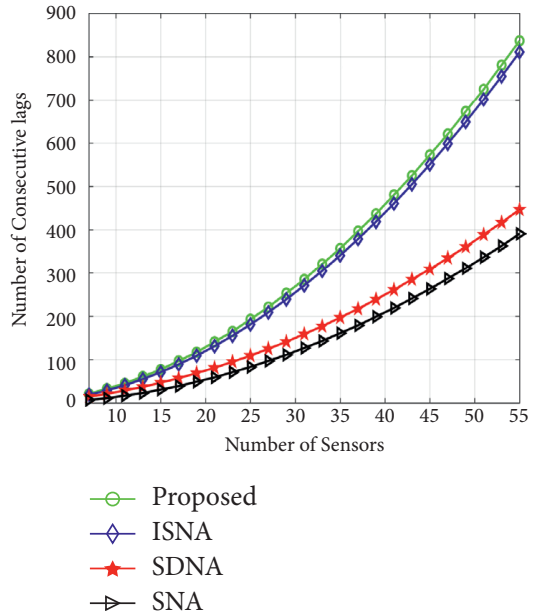

(c)

FIGURE 11: Computational complexity, underdetermined estimate and DOF comparison. (a) Computation complexity for array sensors. (b) Estimate sources versus array sensors. (c) DOF comparison versus different arrays.

from Figure 10(b), in all snapshots, the proposed algorithms can obtain better estimation accuracy than the other algorithms. As is expected, with the increase of snapshot, the RMSE of DOA show a downward trend.

(3) Computational complexity versus array sensors: when array sensors varied from 13 to 25 in 4 steps, from Figure 11(a), we can see that the computational complexity increases with the increase of the number of array sensors, but the computational complexity of the proposed algorithm is still the lowest.
(4) Estimate sources versus array sensors: when snapshots $=250000$, and SNR $=10 \mathrm{~dB}$, fifteen sources uniformly distributed from $-70^{\circ}$ to $70^{\circ}$ were considered. From Figure 11(b), we can see that our proposed array was suitable for underdetermined cases. We used 13 sensors to estimate 15 sources.

(5) DOF comparison versus other arrays: when the array sensors varied from 7 to 55 in 2 steps, from Figure 11(c), we compare the number of DOF achieved from the four arrays. We can see that the number of DOF increases with array sensors, and 
proposed array provided a higher number of DOF than other arrays.

\section{Conclusion}

In this paper, we proposed an enhanced symmetric nested array (ESNA) to solve localization problems about mixed near-field and far-field sources; we also proposed a novel subarray partition method to construct a special cumulant to eliminate the range parameter in the near-field compared with the existing cumulant. By using the ESNA, the effective number of consecutive lags and virtual aperture are increased greatly compared with other arrays. Using a special cumulant, the conventional MUSIC method is used to estimate the mixed sources, and the computation complexity is moderate. Numerical results show that the proposed array has higher resolution ability and provides the improved accuracy of parameter estimation in almost all SNRs levels, and snapshot numbers are compared with the existing arrays. Therefore, the proposed array evidently outperforms the existing arrays for scenarios such as pure NF, pure FF, and mixed sources.

\section{Appendix}

Proof. (a) We prove it step by step:

(1) If $m \in[N, M+N-1], \rho \in[1-N, N-1]$, we have $l_{c 1}(m$, $\rho)=p_{m}-p_{\rho}=(N-1)+2 N(m-N+1)-\rho$. When $m=N$, in the subrange $[2 N, 4 N-2]$, existing $2 N-1$ consecutive lags. When $m \in[N, M+N-1]$, we can get $\mathbb{L}_{1}=$ [2N $(m-N+1), 2 N(m-N+1)+2 N-2]$.

(2) If $m=M+N, \rho \in[N, M+N-1]$, we have $l_{c 2}(m$, $\rho)=2 N(M+N-\rho)-1$. When $\rho=M+N-1$, we can get the lag $2 N-1$. Furthermore, if $\rho \in[N, M+$ $N-1]$, we can obtain the subrange $\mathbb{L}_{2}=[2 N(M+$ $N-\rho)-1]$.

(3) If $m=M+N, p \in[1-N, N-1]$, we have $l_{c 3}(m$, $\rho)=p_{m}-p_{\rho}$, from which we can get $2 N-1$ consecutive lags, corresponding to subrange $\mathbb{L}_{3}=[2 \mathrm{~N}$ $(M+1)-1,2 N(M+2)-3]$.

(4) If $m=M+N-1, \rho=N$, we have $l_{c 4}(m, \rho)=4 N+$ $2 N M-2$, we can have $\mathbb{L}_{4}=4 N+2 N M-2$.

(5) If $m \in[0, N-1], \rho \in[1-N, 0]$, we have $l_{c 5}(m, \rho)=$ $\rho_{m}-p_{\rho}=m-\rho$, in the subrange $\mathbb{L}_{5}=[0,2 N-1]$, existing $2 N-1$ consecutive lags.

When we combine the union of set $\mathbb{L}_{1}$ and set $\mathbb{L}_{2}$, we can get the new set $\mathbb{L}_{12}=\mathbb{L}_{1} \cup \mathbb{L}_{2}, \mathbb{L}_{12}=[2 N(m-N+1)-1,2 N$ $(m-N+1)+2 N-2], m \in[N, M+N-1]$. Consequently, combine the union of the set $\mathbb{L}_{12}, \mathbb{L}_{3}, \mathbb{L}_{4}$, it can be obtained that $4 N+2 M N-1$ consecutive lags in the subrange $[0,4 N+2 M N-2]$.

Similarly, we can get another $4 N+2 M N-1$ consecutive lags in the subrange $[-4 N-2 M N+2,-1]$, when $m \in[-N-M, 0], p \in[-N-M, N-1]$.

Therefore, when range is $[-4 N-2 M N+2,4 N+$ $2 M N-2$ ], we can get $8 N+4 M N-3$ consecutive lags.

\section{Data Availability}

The data used to support the findings of this study are available from the corresponding author upon request.

\section{Conflicts of Interest}

The authors declare that they have no conflicts of interest.

\section{Acknowledgments}

This work was supported in part under Grant No. 62171468 from the National Natural Science Foundation of China.

\section{References}

[1] A. M. Molaei, B. Zakeri, and S. M. Hosseini Andargoli, "A one-step algorithm for mixed far-field and near-field sources localization," Digital Signal Processing, vol. 108, Article ID 102899, 2021.

[2] Z. Zheng, M. Fu, W. Q. Wang, and H. C. So, "Symmetric displaced coprime array configurations for mixed near and far field source localization," IEEE Transactions on Antennas and Propagation, vol. 69, no. 1, 2020.

[3] X. Wu, "Localization of far-field and near-field signals with mixed sparse approach: a generalized symmetric arrays perspective," Signal Processing, vol. 175, Article ID 107665, 2020.

[4] Z. Zheng, Y. Huang, W.-Q. Wang, and H. C. So, "Augmented covariance matrix reconstruction for DOA estimation using difference coarray," IEEE Transactions on Signal Processing, vol. 69, pp. 5345-5358, 2021.

[5] Z. Zheng, M. Fu, W.-Q. Wang, and H. C. So, "Mixed far-field and near-field source localization based on subarray crosscumulant," Signal Processing, vol. 150, pp. 51-56, 2018.

[6] R. Roy and T. Kailath, "Esprit-estimation of signal parameters via rotational invariance techniques," IEEE Transactions on Acoustics, Speech, and Signal Processing, vol. 37, no. 7, 1990.

[7] R. O. Schmidt, "Multiple emitter location and signal parameter estimation," Ph.D. Dissertation, Stanford University, Stanford, CA, USA, 1981.

[8] M. Hyder and K. Mahata, Source Localization and Tracking: A Sparsity-Exploiting Maximum a Posteriori Based Approach, Springer, Berlin, Germany, 2014.

[9] G. Zhao, L. Jie, and F. Shen, "High-resolution DOA estimation with meridian prior," EURASIP Journal on Applied Signal Processing, vol. 2013, no. 1, pp. 1-8, 2013.

[10] E. A. Akbar, A. H. Reza, and K. Mahmood, "Localization of mixed near-field and far-field sources using the largest aperture sparse linear array," IET Signal Processing, vol. 12, no. 2, pp. 155-162, 2018.

[11] Y.-D. Huang and M. Barkat, "Near-field multiple source localization by passive sensor array," IEEE Transactions on Antennas and Propagation, vol. 39, no. 7, pp. 968-975, 1991.

[12] D. Starer and A. Nehorai, "Passive localization of near-field sources by path following," IEEE Transactions on Signal Processing, vol. 42, no. 3, pp. 677-680, 1994.

[13] R. N. Challa and S. Shamsunder, "High-order subspace-based algorithms for passive localization of near-field sources," in Proceedings of the Conference Record of The Twenty-Ninth Asilomar Conference on Signals, Systems and Computers, Pacific Grove, CA, USA, November 1995.

[14] E. Grosicki, K. Abed-Meraim, and Y. Yingbo Hua, "A weighted linear prediction method for near-field source 
localization," IEEE Transactions on Signal Processing, vol. 53, no. 10, pp. 3651-3660, 2005.

[15] W. Zhi and M. Y.-W. Chia, "Near-field source localization via symmetric subarrays," IEEE Signal Processing Letters, vol. 14, no. 6, pp. 409-412, 2007.

[16] B. H. Krim and M. Viberg, "Two decades of array signal processing research," IEEE Signal Processing Magazine, vol. 13, no. 4, 1996.

[17] J. He, M. N. S. Swamy, and M. Omair Ahmad, "Efficient application of music algorithm under the coexistence of farfield and near-field sources," IEEE Transactions on Signal Processing, vol. 60, no. 4, pp. 2066-2070, 2012.

[18] W. Zuo, J. Xin, N. Zheng, A. Sano, Subspace-based localization of far-field and near-field signals without eigendecomposition," IEEE Transactions on Signal Processing, vol. 66, no. $17,2018$.

[19] C. A. Hua, B. Wpz, L. C. Wei et al., "Rare-based localization for mixed near-field and far-field rectilinear sources," Digital Signal Processing, vol. 85, pp. 54-61, 2019.

[20] J. Liang and L. Ding, "Passive localization of mixed near-field and far-field sources using two-stage music algorithm," IEEE Transactions on Signal Processing, vol. 58, no. 1, pp. 108-120, 2009.

[21] Y. Tian, Q. Lian, and H. Xu, "Mixed near-field and far-field source localization utilizing symmetric nested array," Digital Signal Processing, vol. 73, pp. 16-23, 2017.

[22] R. T. Hoctor and S. A. Kassam, "The unifying role of the coarray in aperture synthesis for coherent and incoherent imaging," Proceedings of the IEEE, vol. 78, no. 4, pp. 735-752, 2002.

[23] P. Pal and P. P. Vaidyanathan, "Nested arrays: a novel approach to array processing with enhanced degrees of freedom," IEEE Transactions on Signal Processing, vol. 58, no. 8, pp. 4167-4181, 2010

[24] P. P. Vaidyanathan and P. Pal, "Sparse sensing with co-prime samplers and arrays," IEEE Transactions on Signal Processing, vol. 59, no. 2, pp. 573-586, 2011.

[25] X. Su, P. Hu, Z. Liu, T. Liu, B. Peng, and X. Li, "Mixed nearfield and far-field source localization based on convolution neural networks via symmetric nested array," IEEE Transactions on Vehicular Technology, vol. 70, no. 8, pp. 7908-7920, 2021.

[26] J. He, L. Li, T. Shu, and T. K. Truong, "Mixed near-field and far-field source localization based on exact spatial propagation geometry," IEEE Transactions on Vehicular Technology, vol. 70, no. 4, 2021.

[27] A. M. Molaei, B. Zakeri, and S. Andargoli, "Components separation algorithm for localization and classification of mixed near-field and far-field sources in multipath propagation," IEEE Transactions on Signal Processing, vol. 68, no. 1, pp. 404-419, 2019.

[28] W. Bo, J. Liu, and X. Sun, "Mixed sources localization based on sparse signal reconstruction," Signal Processing Letters, IEEE, vol. 19, no. 8, pp. 487-490, 2012.

[29] M. C. Dogan and J. M. Mendel, "Applications of cumulants to array processing .I. aperture extension and array calibration," IEEE Transactions on Signal Processing, vol. 43, no. 5, 1995.

[30] B. Porat and B. Friedlander, "Direction finding algorithms based on high-order statistics," IEEE Transactions on Signal Processing, vol. 39, no. 9, pp. 2016-2024, 1991.

[31] P. Chevalier, L. Albera, A. Ferreol, and P. Comon, "On the virtual array concept for higher order array processing," IEEE Transactions on Signal Processing, vol. 53, no. 4, pp. 1254-1271, 2005.
[32] Z. Zheng, M. Fu, W.-Q. Wang, S. Zhang, and Y. Liao, "Localization of mixed near-field and far-field sources using symmetric double-nested arrays," IEEE Transactions on Antennas and Propagation, vol. 67, no. 11, pp. 7059-7070, 2019. 